CARPATHIAN J. MATH.

Volume 37 (2021), No. 2,

Pages 195 - 202
Online version at https : //www . carpathian. cunbm . utcluj. ro/

Print Edition: ISSN 1584 - 2851; Online Edition: ISSN 1843 - 4401

DOI: https://doi.org/10.37193/CJM.2021.02.05

Dedicated to Prof. Ioan A. Rus on the occasion of his $85^{\text {th }}$ anniversary

\title{
Functional differential equations with maxima, via step by step contraction principle
}

\author{
VERONICA ILEA*and DiAnA OTROCOL**
}

\begin{abstract}
T. A. Burton presented in some examples of integral equations a notion of progressive contractions on $C([a, \infty[)$. In 2019, I. A. Rus formalized this notion (I. A. Rus, Some variants of contraction principle in the case of operators with Volterra property: step by step contraction principle, Advances in the Theory of Nonlinear Analysis and its Applications, 3 (2019) No. 3, 111-120), put "step by step" instead of "progressive" in this notion, and give some variant of step by step contraction principle in the case of operators with Volterra property on $C([a, b], \mathbb{B})$ and $C([a, \infty[, \mathbb{B})$ where $\mathbb{B}$ is a Banach space. In this paper we use the abstract result given by $\mathrm{I}$. A. Rus, to study some classes of functional differential equations with maxima.
\end{abstract}

\section{INTRODUCTION}

In 1990, Corduneanu investigated functional differential equations involving abstract Volterra operators. In this sense, around the year 2000 Corduneanu [7] presented a general study on functional differential equations with abstract or causal Volterra operators.

On the other hand, T. A. Burton ([3]-[6]) presented in some examples of integral equations a notion of progressive contractions on $C([a, \infty[)$. In 2019, following the idea of T. A. Burton and the forward step method ([19]), I. A. Rus formalized this notion ([21]), with "step by step" instead of "progressive", and give some variant of step by step contraction principle in the case of operators with Volterra property on $C([a, b], \mathbb{B})$ and $C([a, \infty[, \mathbb{B})$ where $\mathbb{B}$ is a Banach space.

In this paper we consider the following functional differential equation with maxima

$$
x^{\prime}(t)=f\left(t, x(t), \max _{a \leq \xi \leq t} x(\xi)\right), t \in[a, b]
$$

with the condition

$$
x(a)=\alpha,
$$

where $\alpha \in \mathbb{R}$ and $f \in C\left([a, b] \times \mathbb{R}^{2}\right)$ are given. To prove our results, we shall use the abstract result given by I. A. Rus [21].

\section{PRELIMINARIES}

2.1. Weakly Picard operators. In the sequel, the following results are useful for some of the proofs in the paper (see [16, 17]).

Let $(X, d)$ be a metric space. An operator $A: X \rightarrow X$ is called weakly Picard operator (WPO) if the sequence of successive approximations, $\left\{A^{n}(x)\right\}_{n \in \mathbb{N}}$, converges for all $x \in X$ and its limit (which generally depend on $x$ ) is a fixed point of $A$. If an operator $A$ is WPO

Received: 26.01.2021. In revised form: 19.04.2021. Accepted: 26.04.2021

2010 Mathematics Subject Classification. 47H10, 47H09, 34K05, 34K12, 45D05, 45G10.

Key words and phrases. G-contraction, step by step contraction, Picard operator, weaakly Picard operator, generalized fibre contraction theorem, functional differential equation, functional integral equation, equation with maxima.

Corresponding author: Veronica Ilea; vdarzu@math.ubbcluj.ro 
with a unique fixed point, that is, $F_{A}=\left\{x^{*}\right\}$, then, by definition, $A$ is called a Picard operator (PO).

If $A: X \rightarrow X$ is a WPO, we can define the operator $A^{\infty}: X \rightarrow F_{A}$, by $A^{\infty}(x):=\lim _{n \rightarrow \infty}$ $A^{n}(x)$.

2.2. $G$-contractions. Let $(X, d)$ be a metric space and $G \subset X \times X$ be a nonempty binary relation. An operator $A: X \rightarrow X$ is a $G$-contraction if there exists $l \in(0,1)$ such that,

$$
d(A(x), A(y)) \leq l d(x, y), \forall(x, y) \in G .
$$

Let us give an example of $G$-contraction. For other examples see [2], [18], [21] and [22].

Let $a<c<b$ and $X:=C[a, b]$, with $d(x, y):=\max _{a \leq t \leq b}|x(t)-y(t)|$. For $H \in C([a, b] \times$ $[a, b] \times \mathbb{R})$ we consider the operator, $A: C[a, b] \rightarrow C[a, b]$ defined by

$$
A(x)(t):=\int_{a}^{t} H\left(t, s, \max _{a \leq \xi \leq s} x(\xi)\right) d s .
$$

We suppose that there exists $L>0$ such that

$$
|H(t, s, u)-H(t, s, v)| \leq L|u-v|, t, s \in[a, b], u, v \in \mathbb{R} .
$$

Let $G:=\left\{(x, y)|x, y \in C([a, b], \mathbb{R}), x|_{[a, c]}=\left.y\right|_{[a, c]}\right\}$. If $L(b-c)<1$, then $A$ is a $G$ contraction.

Indeed for $t \in[a, c]$ if $\left.x\right|_{[a, c]}=\left.y\right|_{[a, c]}$, then $A(x)(t)=A(y)(t)$.

If $t \in[c, b]$, then

$$
\begin{gathered}
A(x)(t)=\int_{a}^{c} H\left(t, s, \max _{a \leq \xi \leq s} x(\xi)\right) d s+\int_{c}^{t} H\left(t, s, \max _{a \leq \xi \leq s} x(\xi)\right) d s \\
x, y \in G \Rightarrow\|A(x)-A(y)\| \leq L(b-c)\|x-y\| .
\end{gathered}
$$

2.3. Step by step contraction. Let $(\mathbb{B},|\cdot|)$ be a (real or complex) Banach space and $C([a, c], \mathbb{B})$ the Banach space with max-norm, $\|\cdot\|$. In what follows, in all spaces of functions we consider max-norm. For $m \in \mathbb{N}, m \geq 2$, let $t_{0}:=a, t_{k}:=t_{0}+k \frac{b-a}{m}, k=\overline{1, m}$. Let $V$ : $C([a, b], \mathbb{B}) \rightarrow C([a, b], \mathbb{B})$ be an operator with Volterra property. Let $V_{k}: C\left(\left[t_{0}, t_{k}\right], \mathbb{B}\right) \rightarrow$ $C\left(\left[t_{0}, t_{k}\right], \mathbb{B}\right), k=\overline{1, m-1}$ the operator induced by $V$ on $C\left(\left[t_{0}, t_{k}\right], \mathbb{B}\right)$. We also consider the following sets,

$$
G_{k}:=\left\{(x, y)\left|x, y \in C\left(\left[t_{0}, t_{k+1}\right], \mathbb{B}\right), x\right|_{\left[t_{0}, t_{k}\right]}=\left.y\right|_{\left[t_{0}, t_{k}\right]}\right\}, k=\overline{1, m-1} .
$$

For $x_{k} \in C\left(\left[t_{0}, t_{k}\right], \mathbb{B}\right), k=\overline{1, m-1}$, we denote

$$
X_{x_{k}}:=\left\{y \in C\left(\left[t_{0}, t_{k+1}\right], \mathbb{B}\right),\left.y\right|_{\left[t_{0}, t_{k}\right]}=x_{k}\right\} .
$$

The following result is given in [21].

Theorem 2.1. (Theorem of step by step contraction). We suppose that:

(1) $V: C([a, b], \mathbb{B}) \rightarrow C([a, b], \mathbb{B})$ has the Volterra property;

(2) $V_{1}$ is a contraction;

(3) $V_{k}$ is a $G_{k-1}$-contraction, for $k=\overline{2, m}$.

Then

(i) $F_{V}=\left\{x^{*}\right\}$; 
(ii)

$$
\begin{aligned}
\left.x^{*}\right|_{\left[t_{0}, t_{1}\right]} & =V_{1}^{\infty}(x), \forall x \in C\left(\left[t_{0}, t_{1}\right], \mathbb{R}\right), \\
\left.x^{*}\right|_{\left[t_{0}, t_{2}\right]} & =V_{2}^{\infty}(x), \forall x \in X_{\left.x^{*}\right|_{\left[t_{0}, t_{1}\right]},} \\
& \vdots \\
\left.x^{*}\right|_{\left[t_{0}, t_{m-1}\right]} & =V_{m-1}^{\infty}(x), \forall x \in X_{\left.x^{*}\right|_{\left[t_{0}, t_{m-2}\right]} ;} ;
\end{aligned}
$$

(iii) $x^{*}=V^{\infty}(x), \quad \forall x \in X_{\left.x^{*}\right|_{\left[t_{0}, t_{m-1}\right]}}$.

For other details and results concerning the theory of $G$-contraction, step by step contraction, Picard operator, weakly Picard Operator and equations with maxima, see: [1], [8]-[21].

\section{MAIN RESULT}

In this section, we shall establish a new result of existence and uniqueness of the solution of the functional differential equation with maxima (1.1).

The problem (1.1)-(1.2), $x \in C^{1}([a, b], \mathbb{R})$ is equivalent with the fixed point equation

$$
x(t)=\alpha+\int_{a}^{t} f\left(s, x(s), \max _{a \leq \xi \leq s} x(\xi)\right) d s, t \in[a, b] .
$$

It is clear that equation (3.3) is equivalent with $x=V(x)$, where the operator $V: C([a, b], \mathbb{R}) \rightarrow$ $C([a, b], \mathbb{R})$, defined by

$$
V(x)(t):=\alpha+\int_{a}^{t} f\left(s, x(s), \max _{a \leq \xi \leq s} x(\xi)\right) d s, t \in[a, b] .
$$

The operator $V$ has the Volterra property, i.e.,

$$
t \in(a, b), x, y \in C[a, b],\left.x\right|_{[a, t]}=\left.\left.y\right|_{[a, t]} \Rightarrow V(x)\right|_{[a, t]}=\left.V(y)\right|_{[a, t]} .
$$

This implies that the operator $V$ induced, for each $c$ with $a<c<b$ and, the operator $V_{c}$ : $C[a, c] \rightarrow C[a, c]$, defined by, $V_{c}(x)(t):=V(\widetilde{x})$, where $\widetilde{x} \in C[a, b]$ is such that, $\left.\widetilde{x}\right|_{[a, c]}=x$.

In what follows we consider the notations from Section 2.3, where $\mathbb{B}=\mathbb{R}$.

We have

Theorem 3.2. We suppose that:

(1) There exists $L>0$, such that

$$
\left|f\left(t, u_{1}, u_{2}\right)-f\left(t, v_{1}, v_{2}\right)\right| \leq L \max \left(\left|u_{1}-v_{1}\right|,\left|u_{2}-v_{2}\right|\right),
$$

for all $t \in[a, b], u_{i}, v_{i} \in \mathbb{R}, i=1,2$.

(2) $m \in \mathbb{N}^{*}$ is such that

$$
\frac{L(b-a)}{m}<1
$$

Then, we have

(i) $F_{V}=\left\{x^{*}\right\}$, i.e., the problem (1.1)-(1.2) has a unique solution. 
(ii)

$$
\begin{aligned}
\left.x^{*}\right|_{\left[t_{0}, t_{1}\right]} & =V_{1}^{\infty}(x), \forall x \in C\left[t_{0}, t_{1}\right], \\
\left.x^{*}\right|_{\left[t_{0}, t_{2}\right]} & =V_{2}^{\infty}(x), \forall x \in X_{x^{*}} \\
& \vdots \\
\left.x^{*}\right|_{\left[t_{0}, t_{m-1}\right]} & =V_{m-1}^{\infty}(x),\left.\forall x \in X_{x^{*}}\right|_{\left[t_{0}, t_{m-1}\right]} .
\end{aligned}
$$

(iii) $x^{*}=V^{\infty}(x),\left.\forall x \in X_{x^{*}}\right|_{\left[t_{0}, t_{m-1}\right]} \cdot$

Proof. We shall prove that in the conditions (1) and choosing $m$ as in (2), we are in the conditions of Theorem of step by step contractions.

Let us prove that $V_{1}$ is an contraction. We have:

$$
\begin{aligned}
\left|V_{1}(x)(t)-V_{1}(y)(t)\right| & \leq\left|\int_{a}^{t} f\left(s, x(s), \max _{a \leq \xi \leq s} x(\xi)\right) d s-\int_{a}^{t} f\left(s, y(s), \max _{a \leq \xi \leq s} y(\xi)\right) d s\right| \\
& \leq L \int_{a}^{t} \max \left(|x(s)-y(s)|,\left|\max _{a \leq \xi \leq s} x(\xi)-\max _{a \leq \xi \leq s} y(\xi)\right|\right) d s \\
& \leq \frac{L(b-a)}{m} \max _{t_{0} \leq t \leq t_{1}}|x(t)-y(t)| .
\end{aligned}
$$

It follows that

$$
\max _{t_{0} \leq t \leq t_{1}}\left|V_{1}(x)(t)-V_{1}(y)(t)\right| \leq \frac{L(b-a)}{m} \max _{t_{0} \leq t \leq t_{1}}|x(t)-y(t)| .
$$

So, $V_{1}$ is a contraction.

Let us prove that $V_{2}$ is a $G_{1}$-contraction. First we remark that, for $t \in\left[t_{0}, t_{1}\right]$

$$
\begin{aligned}
& V_{2}(x)(t)=V_{2}(y)(t), \text { for } x, y \in G_{1} . \\
\left|V_{2}(x)(t)-V_{2}(y)(t)\right|= & \mid \int_{a}^{t_{1}}\left[f\left(s, x(s), \max _{a \leq \xi \leq s} x(\xi)\right)-f\left(s, y(s), \max _{a \leq \xi \leq s} y(\xi)\right)\right] d s \\
& +\int_{t_{1}}^{t}\left[f\left(s, x(s), \max _{a \leq \xi \leq s} x(\xi)\right)-f\left(s, y(s), \max _{a \leq \xi \leq s} y(\xi)\right)\right] d s \mid \\
= & \left|\int_{t_{1}}^{t}\left[f\left(s, x(s), \max _{a \leq \xi \leq s} x(\xi)\right)-f\left(s, y(s), \max _{a \leq \xi \leq s} y(\xi)\right)\right] d s\right| \\
\leq & \frac{L(b-a)}{m} \max _{t_{0} \leq t \leq t_{2}}|x(t)-y(t)| .
\end{aligned}
$$

In a similar way we prove that $V_{3}, \ldots, V_{m}$ are $G_{2}, \ldots, G_{m-1}$ contractions.

Now the prove follows from the Theorem of step by step contractions.

Remark 3.1. In the conditions of the Theorem 3.2 let us denote $\left.x^{*}\right|_{\left[t_{0}, t_{k}\right]}=x_{k}^{*}, 1 \leq k \leq$ $m-1$. Then we have that:

The sequence of successive approximations

$$
x_{1, n+1}(t)=\int_{a}^{t} f\left(s, x_{1, n}(s), \max _{a \leq \xi \leq s} x_{1, n}(\xi)\right) d s, t \in\left[t_{0}, t_{1}\right]
$$

converges uniformly on $\left[t_{0}, t_{1}\right]$ to $x_{1}^{*}=\left.x^{*}\right|_{\left[t_{0}, t_{1}\right]}$. 
The sequence of successive approximations

$$
x_{2, n+1}(t)=\left\{\begin{array}{l}
x_{1}^{*}(t), t \in\left[t_{0}, t_{1}\right] \\
x_{1}^{*}\left(t_{1}\right)+\int_{t_{1}}^{t} f\left(s, x_{2, n}(s), \max _{a \leq \xi \leq s} x_{2, n}(\xi)\right) d s, t \in\left[t_{1}, t_{2}\right]
\end{array}\right.
$$

converges uniformly on $\left[t_{0}, t_{2}\right]$ to $x_{2}^{*}=\left.x^{*}\right|_{\left[t_{0}, t_{2}\right]}$.

...

The sequence of successive approximations

$x_{m-1, n+1}(t)=\left\{\begin{array}{l}x_{m-2}^{*}(t), t \in\left[t_{0}, t_{m-2}\right] \\ x_{m-2}^{*}\left(t_{m-2}\right)+\int_{t_{m-2}}^{t} f\left(s, x_{m-1, n}(s), \max _{a \leq \xi \leq s} x_{m-1, n}(\xi)\right) d s, t \in\left[t_{m-2}, t_{m-1}\right]\end{array}\right.$

converges uniformly on $\left[t_{0}, t_{m-1}\right]$ to $x_{m-1}^{*}=\left.x^{*}\right|_{\left[t_{0}, t_{m-1}\right]}$.

The above considerations give rise to the following problem: In which conditions the operator $V$ is Picard operator?

From the Fibre contraction principle (see [21]) the answer is the following: In the conditions of the Theorem 3.2, the operator $V$ is a Picard operator with respect to the uniform convergence on $\left[t_{0}, t_{m}\right]$.

In order to prove this we consider the following operators induced by the operator $V$. First of all from (3.4) we have that:

(4.1) $V(x)(t):=\alpha+\int_{t_{0}}^{t} f\left(s, x(s), \max _{t_{0} \leq \xi \leq s} x(\xi)\right) d s, t \in\left[t_{0}, t_{1}\right]$,

(4.2) $V(x)(t):=\alpha+\int_{t_{0}}^{t_{1}} f\left(s, x(s), \max _{t_{0} \leq \xi \leq s} x(\xi)\right) d s+\int_{t_{1}}^{t} f\left(s, x(s), \max _{t_{0} \leq \xi \leq s} x(\xi)\right) d s, t \in\left[t_{1}, t_{2}\right]$,

(4.k) $V(x)(t):=\alpha+\int_{t_{0}}^{t_{1}} f\left(s, x(s), \max _{t_{0} \leq \xi \leq s} x(\xi)\right) d s+\ldots+\int_{t_{k-1}}^{t} f\left(s, x(s), \max _{t_{0} \leq \xi \leq s} x(\xi)\right) d s, t \in$ $\left[t_{k-1}, t_{k}\right], k=\overline{1, m}$.

Let $R: C\left[t_{0}, t_{m}\right] \rightarrow C\left[t_{0}, t_{1}\right] \times C\left[t_{1}, t_{2}\right] \times \ldots \times C\left[t_{m-1}, t_{m}\right]$ be defined by,

$$
x \rightarrow\left(\left.x\right|_{\left[t_{0}, t_{1}\right]},\left.x\right|_{\left[t_{1}, t_{2}\right]}, \ldots,\left.x\right|_{\left[t_{m-1}, t_{m}\right]}\right) .
$$

We also consider the following subset:

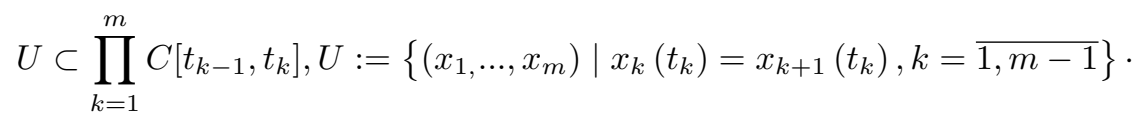

It is clear that $R: C\left[t_{0}, t_{m}\right] \rightarrow U$ is a bijection.

Let us consider the following operators induced by the operator $V$ :

$T_{1}: C\left[t_{0}, t_{1}\right] \rightarrow C\left[t_{0}, t_{1}\right]$

$$
T_{1}\left(x_{1}\right)(t):=\alpha+\int_{t_{0}}^{t} f\left(s, x_{1}(s), \max _{t_{0} \leq \xi \leq s} x_{1}(\xi)\right) d s, t \in\left[t_{0}, t_{1}\right],
$$

$T_{2}: C\left[t_{0}, t_{1}\right] \times C\left[t_{1}, t_{2}\right] \rightarrow C\left[t_{1}, t_{2}\right]$,

$T_{2}\left(x_{1}, x_{2}\right)(t):=\alpha+\int_{t_{0}}^{t_{1}} f\left(s, x_{1}(s), \max _{t_{0} \leq \xi \leq s} x_{1}(\xi)\right) d s+\int_{t_{1}}^{t} f\left(s, x_{2}(s), \max _{t_{0} \leq \xi \leq s} x_{2}(\xi)\right) d s, t \in\left[t_{1}, t_{2}\right]$, 


$$
\begin{aligned}
T_{k}: C\left[t_{0}, t_{1}\right] \times C\left[t_{1}, t_{2}\right] \times & \ldots \times C\left[t_{k-1}, t_{k}\right] \rightarrow C\left[t_{k-1}, t_{k}\right], \\
T_{k}\left(x_{1}, x_{2}, \ldots, x_{k}\right)(t):= & \alpha+\int_{t_{0}}^{t_{1}} f\left(s, x_{1}(s), \max _{t_{0} \leq \xi \leq s} x_{1}(\xi)\right) d s+\ldots \\
& +\int_{t_{k-1}}^{t} f\left(s, x_{k}(s), \max _{t_{0} \leq \xi \leq s} x_{k}(\xi)\right) d s, t \in\left[t_{k-1}, t_{k}\right], k=\overline{1, m}
\end{aligned}
$$

and

$$
T: \prod_{k=1}^{m} C\left[t_{k-1}, t_{k}\right] \rightarrow \prod_{k=1}^{m} C\left[t_{k-1}, t_{k}\right], T:=\left(T_{1}, T_{2}, \ldots, T_{m}\right) .
$$

In the conditions of Theorem 3.2, the operators, $T_{1}, T_{2}\left(x_{1}, \cdot\right), \ldots, T_{m}\left(x_{1}, \ldots, x_{m-1}, \cdot\right)$ are contractions. From the Fibre Contraction Principle, $T$ is a Picard operator.

Now, we observe that: $V=R^{-1} T R$ and $V^{n}=R^{-1} T^{n} R$. These imply that the operator $V$ is a Picard operator.

\section{DifFERENTIAL INEQUALITIES}

In this section we will emphasize the importance of the above result by applying for the operator $V$ the Gronwall type inequalities and the comparison theorem.

In this section we suppose that

$(H)$ there exists $L>0$ such that

$$
\left|f\left(t, u_{1}, u_{2}\right)-f\left(t, v_{1}, v_{2}\right)\right| \leq L \max \left(\left|u_{1}-v_{1}\right|,\left|u_{2}-v_{2}\right|\right)
$$

for all $t \in[a, b]$ and $u_{i}, v_{i} \in \mathbb{R}, i=1,2$.

We consider on $C([a, b], \mathbb{R})$ the max norm and in condition $(H)$, the operator $V$ defined by (3.4) is a Picard operator. So, in the condition $(H)$, the problem (1.1)-(1.2) has in $C([a, b], \mathbb{R})$ a unique solution $x^{*}$. Moreover, for $t \in[a, b], x^{*}(t)=\lim _{n \rightarrow \infty} x_{n}(t)$, for each $x_{0} \in C([a, b], \mathbb{R})$, where $\left(x_{n}\right)_{n \in \mathbb{N}}$ is defined by

$$
x_{n+1}=\alpha+\int_{a}^{t} f\left(s, x_{n}(s), \max _{a \leq \xi \leq s} x_{n}(\xi)\right) d s, t \in[a, b] .
$$

Now we can apply Abstract Gronwall Lemma (see [21]).

Theorem 4.3. Let us consider the problem (1.1)-(1.2) in the condition $(H)$ and $f(t, \cdot, \cdot): \mathbb{R}^{2} \rightarrow \mathbb{R}$ is increasing, i.e., $u_{1} \leq v_{1}, u_{2} \leq v_{2} \Rightarrow f\left(t, u_{1}, u_{2}\right) \leq f\left(t, v_{1}, v_{2}\right)$, for all $t \in[a, b]$. Let us denote by $x^{*}$ the unique solution of (1.1)-(1.2). Then the following implications holds:

(i) $x \in C([a, b], \mathbb{R}), x(a)=\alpha, x^{\prime}(t) \leq f\left(t, x(t), \max _{a \leq \xi \leq t} x(\xi)\right), t \in[a, b] \Rightarrow x \leq x^{*}$;

(ii) $x \in C([a, b], \mathbb{R}), x(a)=\alpha, x^{\prime}(t) \geq f\left(t, x(t), \max _{a \leq \xi \leq t} x(\xi)\right), t \in[a, b] \Rightarrow x \geq x^{*}$.

In a similar way, a comparison theorem for equation (1.1) can be obtained, using the Abstract Comparison Lemma.

We consider now the following functional differential equations with maxima

$$
x^{\prime}(t)=f_{i}\left(t, x(t), \max _{a \leq \xi \leq t} x(\xi)\right), t \in[a, b]
$$

with the condition

$$
x(a)=\alpha_{i},
$$

where $\alpha_{i} \in \mathbb{R}$ and $f_{i} \in C\left([a, b] \times \mathbb{R}^{2}\right), i=1,2,3$ are given. We suppose that 
$\left(H^{\prime}\right)$ there exists $L_{i}>0$ such that

$$
\left|f_{i}\left(t, u_{1}, u_{2}\right)-f_{i}\left(t, v_{1}, v_{2}\right)\right| \leq L_{i} \max \left(\left|u_{1}-v_{1}\right|,\left|u_{2}-v_{2}\right|\right),
$$

for all $t \in[a, b]$ and $u_{1}, v_{1}, u_{2}, v_{2} \in \mathbb{R}, i=1,2,3$.

Theorem 4.4. Let us consider the problems (4.5)-(4.6) in the condition: $\left(H^{\prime}\right), f_{2}(t, \cdot, \cdot): \mathbb{R}^{2} \rightarrow$ $\mathbb{R}$ is increasing, for all $t \in[a, b]$ and $\alpha_{1} \leq \alpha_{2} \leq \alpha_{3}, f_{1} \leq f_{2} \leq f_{3}$. Let us denote by $x_{i}^{*}, i=1,2,3$ the unique solutions of (4.5)-(4.6). Then the following implication holds:

$$
x_{1}(a) \leq x_{2}(a) \leq x_{3}(a) \Rightarrow x_{1}^{*} \leq x_{2}^{*} \leq x_{3}^{*} .
$$

Acknowledgement. The authors would like to express their special thanks and gratitude to Professor Ioan A. Rus for the ideas and continuous support along the years.

\section{REFERENCES}

[1] Bainov, D. D. and Hristova, S., Differential equations with maxima, Chapman \& Hall/CRC Pure and Applied Mathematics, 2011

[2] Berzig, M., Coincidence and common fixed point results on metric spaces endowed with an arbitrary binary relation and applications, J. Fixed Point Theory Appl., 12 (2012), No. 1-2, 221-238

[3] Burton, T. A., Integral equations, transformations, and a Krasnoselskii-Schaefer type fixed point theorem, Electron. J. Qual. Theory Differ. Equ., (2016), No. 66, 1-13; doi: 10.14232/ejqtde.2016.1.66

[4] Burton, T. A., Existence and uniqueness results by progressive contractions for integro- differential equations, Nonlinear Dyn. Syst. Theory, 16 (2016), No. 4, 366-371

[5] Burton, T. A., An existence theorem for a fractional differential equation using progressive contractions, J. Fract. Calc. Appl, 8 (2017), No. 1, 168-172

[6] Burton, T. A., A note on existence and uniqueness for integral equations with sum of two operators: progressive contractions, Fixed Point Theory, 20 (2019), No. 1, 107-112

[7] Corduneanu, C., Abstract Volterra equations: a survey, Math. and Computer Model., 32 (2000), No. (11-13) 1503-1528

[8] Halanay, A., Differential Equations: Stability, Oscillations, Time Lags, Acad. Press, New York, 1966

[9] Ilea, V. and Otrocol, D., On the Burton method of progressive contractions for Volterra integral equations, Fixed Point Theory, 21 (2020), No. 2, 585-594

[10] Marian, D. and Lungu, N., Ulam-Hyers-Rassias stability of some quasilinear partial differential equations of first order, Carpatian J. Math., 35 (2019), No. 2, 165-170

[11] Marian, D., Ciplea, S. A. and Lungu, N., On the Ulam-Hyers stability of biharmonic equation, U. P. B. Sci. Bull., Series A, 82 (2020), No. 2, 141-148

[12] Marian, D., Ciplea, S. A. and Lungu, N., Optimal and nonoptimal Gronwall lemmas, Symmetry, 12 (2020), No. $10,1728,1-10$

[13] Otrocol, D., Ulam stabilities of differential equation with abstract Volterra operator in a Banach space, Nonlinear Funct. Anal. Appl., 15 (2010), No. 4, 613-619

[14] Otrocol, D. and Rus, I. A., Functional-differential equations with "maxima" via weakly Picard operators theory, Bull. Math. Soc. Sci. Math. Roumanie (N. S) , 51 (99) (2008), No. 3, 253-261

[15] Otrocol, D. and Rus, I. A., Functional-differential equations with maxima of mixed type argument, Fixed Point Theory, 9 (2008), No. 1, 207-220

[16] Rus, I. A., Generalized contractions and applications, Cluj University Press, 2001

[17] Rus, I. A., Picard operators and applications, Scientiae Mathematicae Japonicae, 58 (2003), No. 1, 191-219

[18] Rus, I. A., Cyclic representations and fixed points, Ann. T. Popoviciu Seminar of Functional Eq. Approx. Convexity, 3 (2005), 171-178

[19] Rus, I. A., Abstract models of step method which imply the convergence of successive approximations, Fixed Point Theory, 9 (2008), No. 1, 293-307

[20] Rus, I. A., Some nonlinear functional differential and integral equations, via weakly Picard operator theory: a survey, Carpathian J. Math., 26 (2010), No. 2, 230-258

[21] Rus, I. A., Some variants of contraction principle in the case of operators with Volterra property: step by step contraction principle, Advances in the Theory of Nonlinear Analysis and its Applications, 3 (2019), No. 3, 111-120

[22] Samet, B. and Turinici, M., Fixed point theorems on a metric space endowed with an arbitrary binary relation and applications, Commun. Math. Anal., 13 (2012), No. 2, 82-97 
* Department of Mathematics

BABEŞ-BOLYAI UNIVERSITY

FACULTY OF MATHEMATICS AND COMPUTER SCIENCE

M. KogĂLniceanu 1, RO-400084 Cluj-NAPOCA, RoMAniA

Email address: vdarzu@math.ubbcluj.ro

** Technical University of Cluj-Napoca

MEMORANDUMUlui 28, 400114, CluJ-NAPOCA, RomaniA

Tiberiu Popoviciu Institute of Numerical ANAlysis, ROMANiAn ACADEMy

P. O. Box. 68-1, 400110, CLUJ-NAPOCA, ROMANIA

Email address: Diana. Otrocolemath.utcluj.ro 